\title{
Free-form optimization of thin-walled structures for achieving a desired deformed shape
}

\author{
M. Shimoda \\ Department of Advanced Science and Technology, \\ Toyota Technological Institute, Japan
}

\begin{abstract}
Thin-walled structures such as shells and folded plates are extensively used in various industrial products. In this paper, a free-form optimization method is presented that is aimed at giving a function to thin-walled structures. As a concrete target, a method to achieve a desired deformation, or to control a static deformed shape to a desired one, is proposed for the design of linear elastic shell structures. As an objective functional, we introduce a squared error norm of a deformed shape on its prescribed surface. It is assumed that the shell is varied in the normal direction to the surface and that the thickness is constant. A distributed-parameter shape optimization problem is formulated, and the shape gradient function for this problem is theoretically derived. The non-parametric free-form optimization method for shells, which was developed by the author, is applied to solve this problem. With this method, an optimal arbitrarily formed shell with smoothness can be obtained while minimizing the objective functional. The calculated results show the effectiveness of the proposed method for the optimal free-form design of thin-walled structures with a desired deformed shape.

Keywords: optimum design, shape optimization, shell, shape identification, inverse problem, deformation control, traction method.
\end{abstract}

\section{Introduction}

Thin-walled or shell structures have high load-carrying capacity in spite of their thinness and lightness. A smart and simple thin-walled structure may be created by adding a function to them without using any actuators. As such a function, a 
desired deformation, i.e., obtaining a given displacement distribution, against an external force is considered in this study. An optimization technique is necessary for designing such a structure. For executing the shape design of a thin-walled structure with a high level performance yet using the minimum amount of material, it is especially necessary to optimize its curvature distribution while satisfying the design purposes.

The author and colleagues have been developing a free-form optimization method, called the " $\mathrm{H}^{1}$ gradient method for shells" for designing the optimal smooth free-form surface of thin-walled structures with curvatures. In our previous studies, we proposed solutions to stiffness problems [1] and vibration problems [2] of shell structures. Focusing on shape optimization of shell structures, the methods can be categorized into parametric and non-parametric methods in terms of design variables. Although most previously proposed shape optimization methods for shells [3,4] are parametric methods, which require parameterization of the shape in advance and the obtained shape is strongly dominated by the parameterization process, our method is classified as a nonparametric method. The proposed method and its features will be described in the following sections. Another non-parametric method with a filter for smoothing was presented by Bletzinger et al. [5].

In this study, a shape identification problem for linear elastic thin-walled structures is newly solved with the $\mathrm{H}^{1}$ gradient method for shells for the purpose of achieving a desired deformed shape under an external force. Controlling the displacement distribution to a given desired one can contribute to solving stiffness design and compliant design problems of thin-walled structures, which means that the solution described here can impart a function to structures by simply changing their shapes. This design problem is a so-called compliant mechanism design or a homology design, and many related papers proposing topology or shape optimization methods have been published [6-10]. However, few papers have discussed the use of a non-parametric shape optimization method to control the deformation design of thin-walled structures. In this study, the desired deformed shape is identified by introducing a squared error norm of a deformed shape on its prescribed surface as the objective functional. With the free-form optimization method, an optimum thin-walled structure with a smooth free-form surface and a desired deformed shape as its function can be obtained without any shape parameterization.

\section{Governing equation for a shell as a set of infinitesimal flat surfaces}

As shown in Fig. 1(a) and Eqs. (1-3), consider a shell having an initial bounded domain $\Omega \subset \mathbf{R}^{3}$ with the boundary $\partial \Omega$, mid-area $A$ with the boundary $\partial A$, side surface $S$ and thickness $h$. It is assumed for simplicity that a shell structure occupying a bounded domain is a set of infinitesimal flat surfaces as shown in Fig. 1(b).

$$
\Omega=\left\{\left(x_{1}, x_{2}, x_{3}\right) \in \mathbf{R}^{3} \mid\left(x_{1}, x_{2}\right) \in A \subset \mathbf{R}^{2}, x_{3} \in(-h / 2, h / 2)\right\},
$$




$$
\begin{aligned}
& \Omega=A \times\left(-\frac{h}{2}, \frac{h}{2}\right), \\
& S=\partial A \times\left(-\frac{h}{2}, \frac{h}{2}\right)
\end{aligned}
$$

It is assumed that the mapping of the local coordinate system $\left(x_{1}, x_{2}, 0\right)$, which gives the position of the mid-area of the shell, to the global coordinate system $\left(X_{1}, X_{2}, X_{3}\right)$, i.e., $\phi:\left(x_{1}, x_{2}, 0\right) \in \mathbf{R}^{3} \mapsto\left(X_{1}, X_{2}, X_{3}\right) \in \mathbf{R}^{3}$, is piecewise smooth. The Mindlin-Reissner plate theory is applied concerning plate bending, and the coupling of the membrane stiffness and bending stiffness is ignored. Using the sign convention in Fig. 1(b), the displacements expressed by the local coordinates $\boldsymbol{u}=\left\{u_{i}\right\}_{i=1,2,3}$ can be divided into the displacements in the in-plane direction $\left\{u_{\alpha}\right\}_{\alpha=1,2}$ and the displacement in the out-of-plane direction $u_{3}$. In this paper, the subscripts of the Greek letters are expressed as $\alpha=1,2$, and the tensor subscript notation uses Einstein's summation convention and a partial differential notation with respect to the spatial coordinates $(\cdot)_{, i}=\partial(\cdot) / \partial x_{i}$.

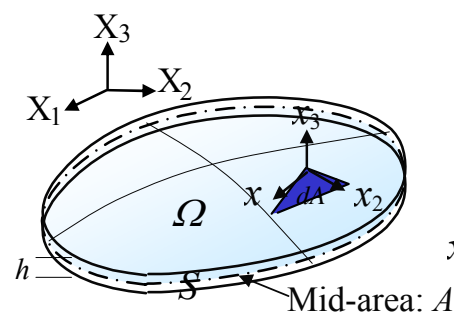

(a) Geometry of a shell

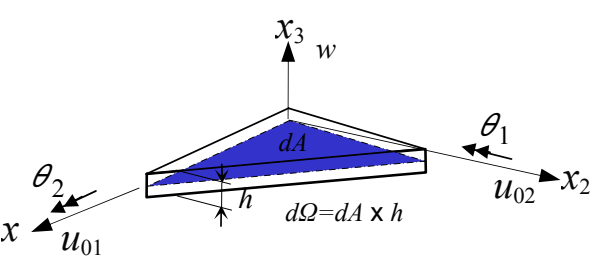

(b) D.O.F. and sign convention

Figure 1: $\quad$ Shell geometry as a set of infinitesimal flat surfaces.

The Mindlin-Reissner plate theory posits that

$$
\begin{gathered}
\sigma_{33}=0, \\
u_{\alpha}\left(x_{1}, x_{2}, x_{3}\right) \equiv u_{0 \alpha}\left(x_{1}, x_{2}\right)-x_{3} \theta_{\alpha}\left(x_{1}, x_{2}\right), \\
u_{3}\left(x_{1}, x_{2}, x_{3}\right) \equiv w\left(x_{1}, x_{2}\right),
\end{gathered}
$$

where $\left\{u_{0 \alpha}\right\}_{\alpha=1,2}, w$ and $\left\{\theta_{\alpha}\right\}_{\alpha=1,2}$ express the in-plane displacements, out-ofplane displacement and rotational angles of the mid-area of the shell, respectively.

Then, the weak form state equation relative to $\left(\boldsymbol{u}_{0}, w, \boldsymbol{\theta}\right) \in U$ can be expressed as Eq. (7) by substituting Eqs. (4-6) into the variational equation for the threedimensional linear elastic body, eliminating $\varepsilon_{33}$. Forces acting relative to the local coordinate system $\left(x_{1}, x_{2}, 0\right)$ on the domain $A$ and the sub-boundary 
$\partial A_{g}(\subset \partial A)$ are defined as follows: an out-of-plane load $q$ per unit area, an inplane loads $\boldsymbol{f}=\left\{f_{\alpha}\right\}_{\alpha=1,2}$ and an out-of-plane moments $\boldsymbol{m}=\left\{m_{\alpha}\right\}_{\alpha=1,2}$ per unit area, an in-plane loads $\boldsymbol{N}=\left\{N_{\alpha}\right\}_{\alpha=1,2}$ per unit length, a shearing force $Q$ per unit length and a bending moments $\boldsymbol{M}=\left\{M_{\alpha}\right\}_{\alpha=1,2}$ per unit length.

$$
a\left(\left(\boldsymbol{u}_{0}, w, \boldsymbol{\theta}\right),\left(\overline{\boldsymbol{u}}_{0}, \bar{w}, \overline{\boldsymbol{\theta}}\right)\right)=l\left(\left(\overline{\boldsymbol{u}}_{0}, \bar{w}, \overline{\boldsymbol{\theta}}\right)\right), \quad\left(\boldsymbol{u}_{0}, w, \boldsymbol{\theta}\right) \in U, \quad \forall\left(\overline{\boldsymbol{u}}_{0}, \bar{w}, \overline{\boldsymbol{\theta}}\right) \in U,
$$

where $\left({ }^{-}\right)$expresses a variation. In addition, the bilinear form $a(\cdot, \cdot)$ and the linear form $l(\cdot)$ are defined respectively as shown below.

$$
\begin{array}{r}
a\left(\left(\boldsymbol{u}_{0}, w, \boldsymbol{\theta}\right),\left(\overline{\boldsymbol{u}}_{0}, \bar{w}, \overline{\boldsymbol{\theta}}\right)\right)=\int_{\Omega}\left\{C_{\alpha \beta \gamma \delta}\left(u_{0 \alpha, \beta}-x_{3} \theta_{\alpha, \beta}\right)\left(\bar{u}_{0 \gamma, \delta}-x_{3} \bar{\theta}_{\gamma, \delta}\right)\right. \\
\left.+C_{\alpha \beta}^{S}\left(w_{, \alpha}-\theta_{\alpha}\right)\left(\bar{w}_{, \beta}-\bar{\theta}_{\beta}\right)\right\} d \Omega, \\
\left(=\int_{A}\left\{c_{\alpha \beta \gamma \delta}^{B} \boldsymbol{\kappa}_{\gamma \delta} \bar{\kappa}_{\alpha \beta}+c_{\alpha \beta \gamma \delta}^{M} u_{0 \gamma, \delta} \bar{u}_{0 \alpha, \beta}+k c_{\alpha \beta}^{S} \gamma_{\alpha} \bar{\gamma}_{\beta}\right\} d A\right) \\
l\left(\left(\overline{\boldsymbol{u}}_{0}, \bar{w}, \overline{\boldsymbol{\theta}}\right)\right)=\int_{A}\left(f_{\alpha} \bar{u}_{0 \alpha}-m_{\alpha} \bar{\theta}_{\alpha}+q \bar{w}\right) d A+\int_{\partial A_{g}}\left(N_{\alpha} \bar{u}_{0 \alpha} d s-M_{\alpha} \bar{\theta}_{\alpha}+Q \bar{w}\right) d s,
\end{array}
$$

where $\left\{C_{\alpha \beta \gamma \delta}\right\}_{\alpha, \beta, \gamma, \delta=1,2}$ and $\left\{C_{\alpha \beta}^{S}\right\}_{\alpha, \beta=1,2}$ express an elastic tensor including bending and membrane stresses, and an elastic tensor with respect to the shearing stress, respectively. $\left\{c_{\alpha \beta \gamma \delta}^{B}\right\}_{\alpha, \beta, \gamma, \delta=1,2},\left\{c_{\alpha \beta}^{S}\right\}_{\alpha, \beta=1,2}$ and $\left\{c_{\alpha \beta \gamma \delta}^{M}\right\}_{\alpha, \beta, \gamma, \delta=1,2}$ express an elastic tensor with respect to bending, shearing and membrane component in case of considering the relationship $\int_{\Omega}(\cdot) d \Omega=\int_{A} \int_{-h / 2}^{h / 2}(\cdot) d z d A$, respectively. In addition, $\left\{\kappa_{\alpha \beta}\right\}_{\alpha, \beta=1,2}$ and $\left\{\gamma_{\alpha}\right\}_{\alpha=1,2}$ express the curvatures and the transverse shear strains which are defined by the following equations. The constant $k$ denotes a shear correction factor (assuming $k=5 / 6$ ).

$$
\begin{gathered}
\kappa_{\alpha \beta} \equiv \frac{1}{2}\left(\theta_{\alpha, \beta}+\theta_{\beta, \alpha}\right), \\
\gamma_{\alpha} \equiv w_{, \alpha}-\theta_{\alpha} .
\end{gathered}
$$

It will be noted that $U$ in Eq. (7) is given by the following equation.

$$
U=\left\{\left(u_{01}, u_{02}, w, \theta_{1}, \theta_{2}\right) \in\left(H^{1}(A)\right)^{5} \mid \begin{array}{cr}
\text { satisfy the given Dirichlet condition on each } \\
\text { subboundary }\},
\end{array}\right.
$$

where $H^{1}$ is the Sobolev space of order 1 . 


\section{Free-form optimization problem of shell structure}

\subsection{Domain variation}

Consider that a linear elastic shell structure having an initial domain $\Omega$, midarea $A$, boundary $\partial A$ and side surface $S$ undergoes domain variation $\boldsymbol{V}$ (design velocity field) in the out-of-plane direction such that its domain, mid-area, boundary and side surface become $\Omega_{s}, A_{s}, \partial A_{s}$ and $S_{s}$, respectively as shown in Fig. 2. It is assumed that the plate thickness $h$ remains constant under the domain variation. The domain variation at this time can be expressed by a mapping from $\Omega$ to $\Omega_{s}$, which is denoted as $T_{S}: \boldsymbol{X} \in \Omega \mapsto \boldsymbol{X}_{s}(\boldsymbol{X}) \in \Omega_{s}, 0 \leq s \leq \varepsilon \quad(\varepsilon \quad$ is a small integer $)$ given by $\boldsymbol{X}_{s}=T_{s}(\boldsymbol{X}), \Omega_{s}=T_{s}(\Omega)$. The subscript $s$ expresses the iteration history of the domain variation. Assuming a shape constraint is acting on the variation in the domain, the infinitesimal variation of the domain can be expressed by

$$
T_{s+\Delta s}(\boldsymbol{X})=T_{s}(\boldsymbol{X})+\Delta s \boldsymbol{V},
$$

where the design velocity field is given as $\boldsymbol{V}\left(\boldsymbol{X}_{s}\right)=\partial T_{s}(\boldsymbol{X}) / \partial s$. The free-form optimization method explained later is a method for determining the optimal domain variation $\boldsymbol{V}$ of shell structures.

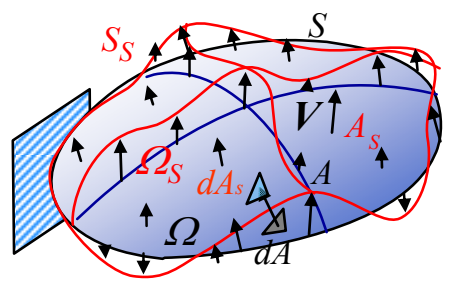

Figure 2: $\quad$ Out-of-plane shape variation $\boldsymbol{V}$.

\subsection{Shape identification problem for achieving a desired deformed shape}

Let us consider a free-form optimization problem for achieving a given desired deformed shape of a thin-walled structure. This problem is formulated in a function space, and the shape gradient function is theoretically derived using the material derivative method as described below.

As an objective functional, we introduce a squared deformed shape error norm on a prescribed surface. Letting the state equation in Eq. (7) be the constraint condition, a distributed-parameter shape identification problem for finding the optimal design velocity field $\boldsymbol{V}$, or $A_{s}(=A+\Delta s \boldsymbol{V})$ can be formulated as shown below:

$$
\text { Given } A
$$




$$
\begin{aligned}
\qquad \begin{array}{c}
\text { Find } \\
\text { that mimimizes }
\end{array} & d\left(\left(\left(\boldsymbol{x}_{0}+\boldsymbol{u}\right)-\hat{\boldsymbol{z}}\right),\left(\left(\boldsymbol{x}_{0}+\boldsymbol{u}\right)-\hat{\boldsymbol{z}}\right)\right) \\
\text { subject to } & \text { Eq. (7), }
\end{aligned}
$$

where $d(\bullet, \bullet)$ is the inner product defined as shown in the following equation.

$$
d(\boldsymbol{u}, \boldsymbol{v})=\int_{A_{D}} u_{i} v_{i} d A
$$

The notations $\boldsymbol{x}_{0}+\boldsymbol{u}$ and $\hat{\boldsymbol{z}}$ indicate the position vector of the deformed shape and that of a given desired deformed shape, respectively, which are given on the prescribed surface $A_{D}$.

Letting $\left(\overline{\boldsymbol{u}}_{0}, \bar{w}, \overline{\boldsymbol{\theta}}\right)$ denote the Lagrange multipliers for the state equation, the Lagrange functional $L$ for this problem can be expressed as

$$
\begin{aligned}
L\left(A,\left(\boldsymbol{u}_{0}, w, \boldsymbol{\theta}\right),\left(\overline{\boldsymbol{u}}_{0}, \bar{w}, \overline{\boldsymbol{\theta}}\right)\right)= & d\left(\left(\boldsymbol{x}_{0}+\boldsymbol{u}-\hat{\boldsymbol{z}}\right),\left(\boldsymbol{x}_{0}+\boldsymbol{u}-\hat{\boldsymbol{z}}\right)\right) \\
& +l\left(\overline{\boldsymbol{u}}_{0}, \bar{w}, \overline{\boldsymbol{\theta}}\right)-a\left(\left(\boldsymbol{u}_{0}, w, \boldsymbol{\theta}\right),\left(\overline{\boldsymbol{u}}_{0}, \bar{w}, \overline{\boldsymbol{\theta}}\right)\right)
\end{aligned}
$$

For the sake of simplicity, it is assumed that the sub-boundaries acted on by the non-zero external forces $\boldsymbol{N}, \boldsymbol{Q}$ and $\boldsymbol{M}$ do not vary (i.e., $\boldsymbol{V}=0$ ), and that the forces acting on the shell surface $\boldsymbol{f}, \boldsymbol{m}, q$ do not vary with regard to the space and the iteration history $s$ (i.e., $(\dot{f}=\dot{m}=\dot{q}=0)$. Then, the material derivative $\dot{L}$ of the Lagrange functional can be derived as shown in Eq. (20) below using the formula of material derivative [12].

Letting $\boldsymbol{n}^{\text {mid }}(\equiv \boldsymbol{n})=\boldsymbol{n}^{\text {top }}=-\boldsymbol{n}^{\text {btm }}$ represent a unit normal vector of the midarea, the relationship $\left(\boldsymbol{V} \cdot \boldsymbol{n}^{t o p}\right) \boldsymbol{n}^{\text {top }}=-\left(\boldsymbol{V} \cdot \boldsymbol{n}^{b t m}\right) \boldsymbol{n}^{b t m}$ is assumed. The notations $\boldsymbol{n}^{\text {top }}$ and $\boldsymbol{n}^{\text {btm }}$ denote unit normal vectors that make the outward top and bottom surfaces of the shell positive. The notations $(\cdot)^{\prime}$ and $(\cdot)$ are the shape derivative and the material derivative with respect to the domain variation, respectively [12].

$$
\begin{gathered}
\dot{L}=2 d\left(\boldsymbol{x}_{0}+\boldsymbol{u}-\hat{\boldsymbol{z}}, \boldsymbol{u}^{\prime}\right)-a\left(\left(\boldsymbol{u}_{0}^{\prime}, w^{\prime}, \boldsymbol{\theta}^{\prime}\right),\left(\overline{\boldsymbol{u}_{0}}, \bar{w}, \overline{\boldsymbol{\theta}}\right)\right) \\
+l\left(\overline{\boldsymbol{u}}_{0}^{\prime}, \bar{w}^{\prime}, \overline{\boldsymbol{\theta}}^{\prime}\right)-a\left(\left(\boldsymbol{u}_{0}, w, \boldsymbol{\theta}\right),\left(\overline{\boldsymbol{u}}_{0}^{\prime}, \bar{w}^{\prime}, \overline{\boldsymbol{\theta}}^{\prime}\right)\right)+\langle G \boldsymbol{n}, \boldsymbol{V}\rangle, \quad \boldsymbol{V} \in C_{\Theta} \\
\langle G \boldsymbol{n}, \boldsymbol{V}\rangle \equiv \int_{A} G \boldsymbol{n} \cdot V d A+\int_{A_{D}} G_{D} \boldsymbol{n} \cdot V d A=\int_{A} \boldsymbol{G} \cdot V d A+\int_{A_{D}} \boldsymbol{G}_{\boldsymbol{D}} \cdot V d A \\
G=-\left\{C_{\alpha \beta \gamma \delta}\left(u_{0 \alpha, \beta}+\frac{h}{2} \theta_{\alpha, \beta}\right)\left(\bar{u}_{0 \gamma, \delta}+\frac{h}{2} \bar{\theta}_{\gamma, \delta}\right)-C_{\alpha \beta \gamma \delta}\left(u_{0 \alpha, \beta}-\frac{h}{2} \theta_{\alpha, \beta}\right)\left(\bar{u}_{0 \gamma, \delta}-\frac{h}{2} \bar{\theta}_{\gamma, \delta}\right)\right. \\
+H f_{\alpha} \bar{u}_{0 \alpha}-H m_{\alpha} \bar{\theta}_{\alpha}+H q \bar{w}
\end{gathered}
$$

Equations (22) and (23) express the shape gradient functions, i.e., the sensitivity functions, for this problem. The notation $H$ denotes twice the mean 
curvature of the surface. $C_{\Theta}$ expresses the kinematically admissible function space that satisfies the constraints of shape variation. When the optimality conditions with respect to the state variable $\left(\boldsymbol{u}_{0}, w, \boldsymbol{\theta}\right)$, the adjoint variable $\left(\overline{\boldsymbol{u}}_{0}, \bar{w}, \overline{\boldsymbol{\theta}}\right)$ of the Lagrange functional $L$ expressed by Eqs. (24) and (25), are satisfied,

$$
\begin{gathered}
a\left(\left(\boldsymbol{u}_{0}, w, \boldsymbol{\theta}\right),\left(\overline{\boldsymbol{u}}_{0}^{\prime}, \bar{w}^{\prime}, \overline{\boldsymbol{\theta}}^{\prime}\right)\right)=l\left(\left(\overline{\boldsymbol{u}}_{0}^{\prime}, \bar{w}^{\prime}, \overline{\boldsymbol{\theta}}^{\prime}\right)\right), \quad \forall\left(\overline{\boldsymbol{u}}_{0}^{\prime}, \bar{w}^{\prime}, \overline{\boldsymbol{\theta}}^{\prime}\right) \in U \\
a\left(\left(\boldsymbol{u}_{0}^{\prime}, w^{\prime}, \boldsymbol{\theta}^{\prime}\right),\left(\overline{\boldsymbol{u}_{0}}, \bar{w}, \overline{\boldsymbol{\theta}}\right)\right)=2 d\left(\boldsymbol{x}_{0}+\boldsymbol{u}-\hat{\boldsymbol{z}}, \boldsymbol{u}^{\prime}\right), \quad \forall\left(\boldsymbol{u}_{0}^{\prime}, w^{\prime}, \boldsymbol{\theta}^{\prime}\right) \in U
\end{gathered}
$$

Eq. (20) becomes

$$
\dot{L} \equiv\langle G \boldsymbol{n}, \boldsymbol{V}\rangle, \quad \boldsymbol{V} \in C_{\Theta} .
$$

Equation (24) is the governing equation for the state variable $\left(\boldsymbol{u}_{0}, w, \boldsymbol{\theta}\right)$ that coincides with Eq. (7), and Eq. (25) is the governing equation for the adjoint variable $\left(\overline{\boldsymbol{u}}_{0}, \bar{w}, \overline{\boldsymbol{\theta}}\right)$.

The shape gradient function derived is applied to the free-form optimization method for shells.

\section{Free-form optimization method $\left(\mathrm{H}^{1}\right.$ gradient method for shells) for designing the optimal surface of thin-walled structures}

The free-form optimization method developed by the author is based on the traction method (often called the $\mathrm{H}^{1}$ gradient method), which is a gradient method in a Hilbert space. The original $\mathrm{H}^{1}$ gradient method was proposed by Azegami in 1994 [11]. It is a non-parametric shape optimization method that can treat all nodes as design variables and does not require any design variable parameterization. The original method has been modified for shells by the author, and called the free-form optimization method or the $\mathrm{H}^{1}$ gradient method for shells. This method varies a shell in the normal direction to the surface, making it possible to obtain the optimal free-form shape of shell structures.

As shown in Fig. 3, a distributed force proportional to the shape gradient function $-\boldsymbol{G}$ is applied in the normal direction to the surface in order to vary the surface. The Robin boundary condition (spring constant $\alpha>0$ ) is defined for the pseudo-elastic shell. This analysis for shape variation is called a velocity analysis. As the shape gradient function is not used directly to vary the shape but rather is replaced to a distributed force, this makes it possible both to reduce the objective functional and to maintain the smoothness, i.e., mesh regularity, which is the most distinctive feature of this method. The displacements obtained as the optimum shape variation in the velocity analysis are added to the original shape to update iteratively the shape. Considering the design velocity $V=\left\{V_{i}\right\}_{i=1,2,3}$ as a combination of the in-plane velocity $\left\{\boldsymbol{V}_{0_{\beta}}\right\}_{\beta=1,2}$ and the out-of-plane velocity $V_{3}$, which are defined in local coordinate systems, the governing equation of the 
velocity analysis for $V=\left(V_{0_{1}}, V_{0_{2}}, V_{3}\right)$ is expressed as Eq. (27) with the definition of $C_{\Theta}$, Eq. (28). Equation (27) can be solved using a standard finite element code.
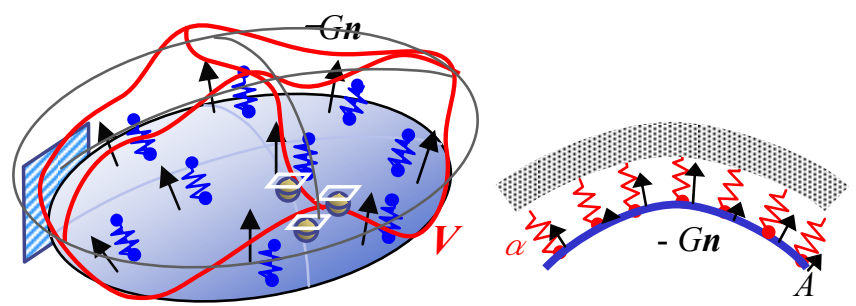

Figure 3: $\quad$ Schematic of free-form optimization method (" $\mathrm{H}^{1}$ gradient method for shell" with Robin condition).

$$
\begin{gathered}
a\left(\left(\boldsymbol{V}_{0 \alpha}, V_{3}, \boldsymbol{\theta}\right),\left(\overline{\boldsymbol{u}}_{0}, \bar{w}, \overline{\boldsymbol{\theta}}\right)\right)+\alpha\left\langle(\boldsymbol{V} \cdot \boldsymbol{n}) \boldsymbol{n},\left(\overline{\boldsymbol{u}}_{0}, \bar{w}, \overline{\boldsymbol{\theta}}\right)\right\rangle=-\left\langle G \boldsymbol{n},\left(\overline{\boldsymbol{u}}_{0}, \bar{w}, \overline{\boldsymbol{\theta}}\right)\right\rangle, \\
\left(\boldsymbol{V}_{0 \alpha}, V_{3}, \boldsymbol{\theta}\right) \in C_{\Theta}, \quad \forall\left(\overline{\boldsymbol{u}}_{0}, \bar{w}, \overline{\boldsymbol{\theta}}\right) \in C_{\Theta}, \\
C_{\Theta}=\left\{\left(V_{0_{1}}, V_{0_{2}}, V_{3}, \theta_{1}, \theta_{2}\right) \in\left(H^{1}(A)\right)^{5} \mid\right.
\end{gathered}
$$

It is confirmed that this gradient method in a Hilbert spaces reduces the Lagrange functional $L$ as follows. When the state equation and the adjoint equation are satisfied, the perturbation expansion of the Lagrange functional $L$ can be written as

$$
\Delta L=\langle G \boldsymbol{n}, \Delta s(\boldsymbol{V}, \boldsymbol{\theta})\rangle+O(|\Delta s|) .
$$

Substituting Eq. (27) into Eq. (29) and taking into account the positive definitiveness of $a\left(\left(\boldsymbol{V}_{0 \alpha}, V_{3}, \boldsymbol{\theta}\right),\left(\overline{\boldsymbol{u}}_{0}, \bar{w}, \overline{\boldsymbol{\theta}}\right)\right)$ and $\alpha\left\langle(\boldsymbol{V} \cdot \boldsymbol{n}) \boldsymbol{n},\left(\overline{\boldsymbol{u}}_{0}, \bar{w}, \overline{\boldsymbol{\theta}}\right)\right\rangle$, based on the positive definitiveness of the elastic tensors $C_{\alpha \beta \gamma \delta}$ and $C_{\alpha \beta}^{S}$, the following relationship is obtained when $\Delta s$ is sufficiently small:

$$
\Delta L=-a(\Delta s(\boldsymbol{V}, \boldsymbol{\theta}), \Delta s(\boldsymbol{V}, \boldsymbol{\theta}))-\alpha\langle(\Delta s \boldsymbol{V} \cdot \boldsymbol{n}) \boldsymbol{n}, \Delta s(\boldsymbol{V}, \boldsymbol{\theta})\rangle<0
$$

In problems where convexity is assured, this relationship definitely reduces the Lagrange functional in the process of updating the shell shape using the design velocity field $\boldsymbol{V}$ determined by Eq. (27).

The advantages offered by this method are summarized as follows: (1) a smooth and natural surface without any jaggedness can be obtained because the elastic tensor in the velocity analysis serves as a mapping function and as a smoother for maintaining mesh smoothness, and its positive definitiveness is the 
necessary condition for minimizing the objective functional. (2) An optimal freeform surface is created because the number of the design degrees of freedom is not limited. (3) It does not require shape design parameterization unlike the basis vector method or the parametric surface method because all the nodes can be moved as the design variable. (4) Mesh smoothing is simultaneously implemented in the shape changing process. (5) It can be easily implemented in combination with a commercial FEA code, which means it has generality and practical utility for actual design work. (6) It is not necessary to refine the mesh.

\section{Calculated results obtained with free-form optimization method}

The proposed method was applied to three fundamental design examples in order to verify its validity for controlling the deformed shape of a thin-walled structure to a desired shape.

\subsection{Roof problem 1}

Problem definition 1 for a cylindrical roof model is shown in Fig. 4. In the stiffness analysis Fig. 4(a), the bottom edges of the roof were simply supported and the downward nodal forces were applied along the line on the top. The deformed shape region was prescribed in the portion around the loaded line on the top as shown in Fig. 4(a). The desired deformed shape was defined as one in which the prescribed region was uniformly deformed downward as shown in Fig. 4(c). In the velocity analysis, the bottom edges were simply supported as shown in Fig. 4(b).

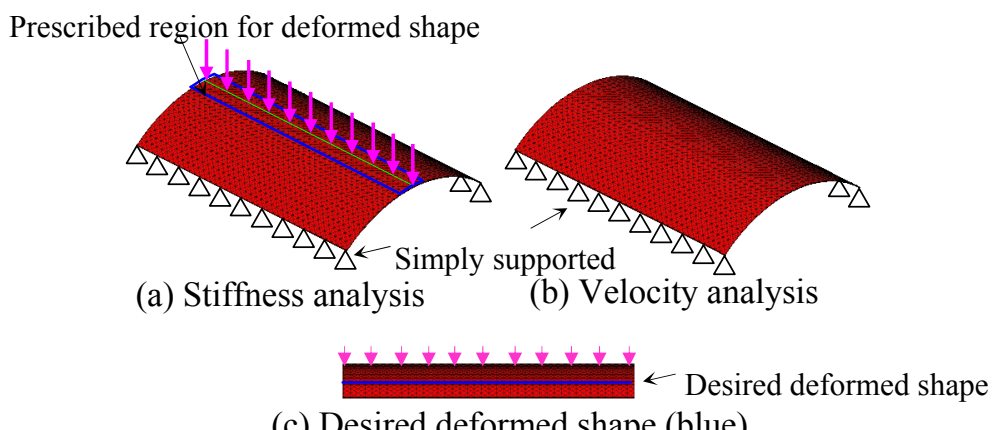

Figure 4: $\quad$ Problem definition for roof problem 1.

The optimal shape obtained and the iteration convergence histories are shown in Fig. 5(a) and (b), respectively. It is seen in Fig. 5(a) that both edges on the top were folded upward for stiffening the edges. The results in Fig. 5(b) indicate that the objective functional converged almost to zero. As a volume constraint was not defined in this study, the calculated results show that the initial volume was 
kept almost constant. Figure 6 compares the deformed shapes between (a) the initial and (b) the final. The figures show that although the region of the initial shape was not deformed uniformly, the region of the final shape was deformed downward uniformly as desired.

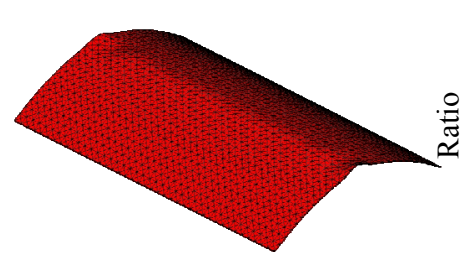

(a) Obtained shape

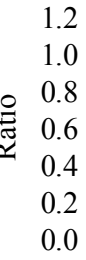

0.0

0

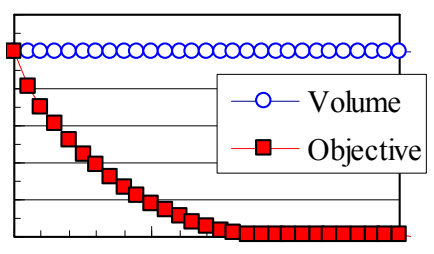

10

20

No. of Iterations

(b) Iteration histories

Figure 5: $\quad$ Calculated results for roof problem 1.

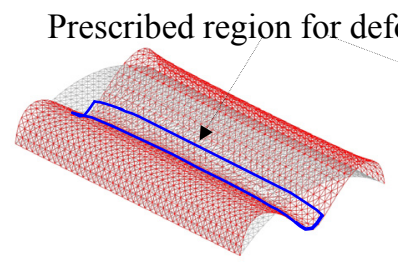

(a) Initial

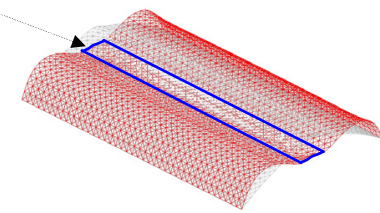

(b) Final

Figure 6: $\quad$ Comparison of deformed shapes of roof problem 1.

\subsection{Roof problem 2}

The problem definition of roof problem 2 is shown in Fig. 7 using the same model as in roof problem 1 except for the prescription of the deformed shape. The loaded line on the top was defined as the prescribed positions of the deformed shape as shown in Fig. 7(a). The desired deformed shape was defined as one in which the line around half of the top maintained the position and was folded downward linearly at the three-quarters point from the top edges as shown in Fig. 7(c). 


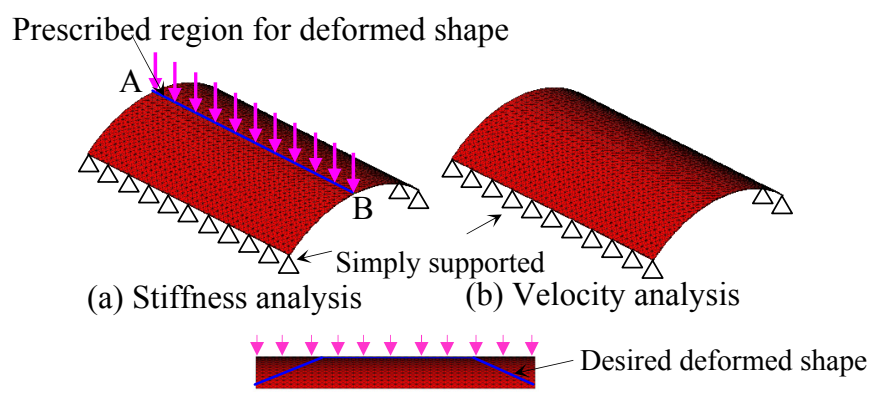

(c) Desired deformed shape (blue)

Figure 7: $\quad$ Problem definition for roof problem 2.

Figure 8(a) shows the optimal shape obtained. It is seen that the portions around the edges of the top were linearly varied downward according to the desired deformed shape, which is reasonable for the prescription. Figure 8(b) compares the deformed positions along the prescribed line for the initial, desired and final shapes. The graph indicates that the deformed positions of the final shape coincided well with the desired positions as intended.

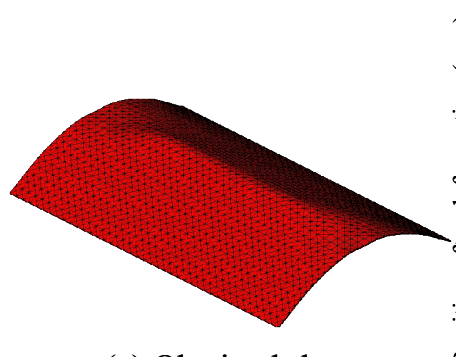

(a) Obtained shape

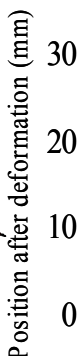

(b) Comparison of deformed shape

Figure 8: Calculated results of roof problem 2.

\subsection{Table problem}

A table problem is defined in Fig. 9. The initial shape and the boundary condition of the stiffness analysis are shown in (a). The bottom edges were simply supported and the top surface was uniformly loaded downward in the stiffness analysis. The bottom edges were also simply supported in the velocity analysis. As shown in Fig. 9(b), the prescribed square regions of the deformed shape were defined in the centres of both side surfaces, and the target shape was defined as one in which the initial positions were kept. 


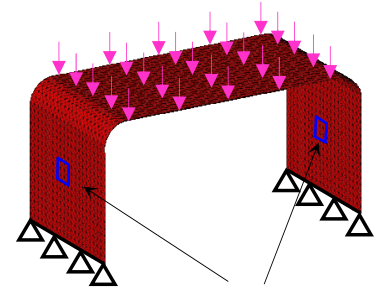

Prescribed region for deformed shape

(a) Stiffness analysis

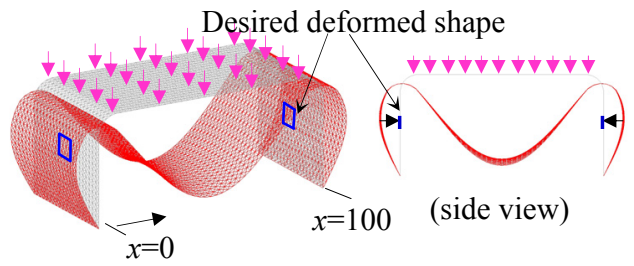

(b) Deformation of initial shape

Figure 9: $\quad$ Problem definition for table problem.

Figure 10(a) shows the optimal shape obtained. Several beads were created on both side surfaces to increase the stiffness. Figure 10(b) shows the deformed shapes of the obtained shape. It was confirmed that the prescribed regions of the final shape kept their positions, although those of the initial shape largely deformed outward.

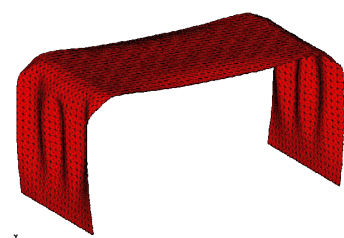

(a) Obtained shape

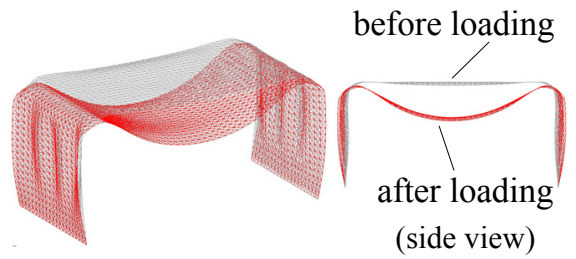

(b) Deformation of obtained shape

Figure 10: Obtained shape and deformation of table problem.

\section{Conclusion}

This paper has presented a shell free-form optimization method for controlling a static deformed shape to a desired shape. A shape identification problem, in which the squared error norm of the deformed shape was defined as the objective functional, was formulated as a distributed-parameter shape optimization problem. The shape gradient function with respect to the shape variation in the normal direction to the shell surface was derived theoretically and applied to the $\mathrm{H}^{1}$ gradient method for shells. With this method, the objective functional converged almost to zero in all the design examples, and shell shapes with beads coinciding with the prescribed deformed shape were obtained. It was confirmed that the use of this method makes it possible to control the deformed shape to a desired shape and imparts a function to thin-walled structures, while creating .an optimal arbitrarily formed surface. 


\section{Acknowledgements}

This research was supported by grants-in-aid from the Sustainable Mechanical Systems R\&D Centre at the Toyota Technological Institute.

\section{References}

[1] M. Shimoda, K. Iwasa and H. Azegami, A Shape optimization method for the optimal free-form design of shell structures, in Proceedings of 8th World Congress on Structural and Multidisciplinary Optimization (WCSMO-8), H.C. Rodrigues, J. M. Guedes, et al. (eds), (2009, Lisbon).

[2] M. Shimoda, Optimal free-form design of shell structures involving a natural frequency problem, in Proceedings of EUROGEN2011 Conference (Evolutionary And Deterministic Methods For Design, Optimization And Control), C. Poloni, D. Quagliarella, et al. (eds.), (2011, Capua).

[3] E. Ramm, K.-U. Bletzinger and R. Reitiger, Shape optimization of shell structures, Int. J. Shell and Spatial Structures, 34 (2), pp.103-121, 1993.

[4] T. Lindby and J. L. T. Santos, Shape Optimization of three-dimensional shell structures with the shape parameterization of a CAD system, Structural Optimization, 18, pp.126-133, 1999.

[5] K. U. Bletzinger, M. Firl, et al., Optimal shapes of mechanically motivated surfaces, Comput. Methods Appl. Mech. Engrg., 199, pp. 324-333, 2010.

[6] N. Yoshikawa and S. Nakagiri, Homology design to maintain quadratic curves, Proc. 5-th Int. Conf. on Adaptive Structures, pp.1-9, (1994, Sendai).

[7] S. Nishiwaki, M. I. Frecker, et al., Topology optimization of compliant mechanisms using the homogenization method, Int. J. of Num. Meth. Engrg., 42 (3), pp.535-560, 1998.

[8] L. Saggere and S. Kota, Static shape control of smart structures using compliant mechanisms, AIAA J. 37 (5), pp.572-579, 1999.

[9] K. J. Lu and S. Kota, Design of compliant mechanisms for morphing structural shapes, J. of Intelligent Material Systems and Structures, 14 (6), pp.379-391, 2003.

[10] M. Shimoda, H. Azegami and T. Sakurai, Traction method approach to optimal shape design problems, SAE Transactions, Journal of Passenger Cars, 6 (106), pp.2355-2365, 1998.

[11] H. Azegami, A Solution to domain optimization problems, Trans. of Jpn. Soc. of Mech. Engs., Ser. A, 60, pp.1479-1486, 1994 (in Japanese).

[12] K. K. Choi and N. H. Kim, Structural Sensitivity Analysis and Optimization, 1, Springer, New York, 2005. 